\title{
REPROCESSING OF UV AND LINE EMISSION IN AGN ACCRETION DISCS
}

\author{
EVLABIA ROKAKI \\ QMW, Univ. of London, Dept of Physics, Mile End Road, London E1 4NS, UK \\ and \\ CATHERINE BOISSON \\ DAEC, Observatoire de Paris-Meudon, Meudon, France
}

It is commonly admitted that AGN contain a massive black hole fuelled most likely by an accretion disc. Several spectral features of the AGN, as the continuum excess in the UV and the broad line spectrum, involving different physical processes of emission (thermal for the UV continuum, photoionisation for the line spectrum) have been proposed as signatures of the disc. Physical parameters of the nucleus (as the mass of the black hole, $M$, the disc inclination, $i$, and accretion rate, $\dot{M}$ ) are better determined when these two spectral features are modelled simultaneously. Here, we present results from the disc modelling (see [1]) of the UV and broad $\mathrm{H} \beta$ emission of the 22 Seyfert 1 galaxies in a complete AGN sample selected in a hard X-ray survey [2].

The comparison of the Eddington, $L_{E d d}\left(=1.2610^{38} \mathrm{M} / M_{\odot} \mathrm{erg} \mathrm{s}^{-1}\right)$ and bolometric, $L_{B o l}$, luminosities shows that all objects radiate well below the Eddington limit $\left(L_{B o}=\right.$ $\left.(0.2-2.5) 10^{-1} L_{E d d}\right)$. The derived values of $i$ span the range of $15^{\circ}-45^{\circ}$ and are consistent with the AGN unified scheme for the Seyferts 1 nuclei. An interesting parameter, derived from the line fitting is also the size of the region which emits the bulk of the line emission $R_{H \beta}$. This parameter (multiplied by $c$ ) represents the time lag of the line light curve with respect to that of the continuum. A relation $R_{H \beta} \propto L_{B o l}^{0.53}$ is derived.

The contribution of the UV emission of the disc (gravitationally heated), $L_{U V}$, to the overall emission of the nucleus is significantly scattered and this is most likely to be due to the heavy absorption of the UV emission from some objects in the sample. When these objects are excluded the disc contribution seems independent of the bolometric luminosity with $L_{U V} \sim 0.15 L_{B o}$.

For the non-absorbed objects we also compute the UV spectra assuming both radiative, (X-ray) and gravitational heating of the disc as is described in [3]. The $\mathrm{X}$-ray source is assumed spherical with a radius equal to the inner radius of the disc $\left(=3 R_{g r}\right)$, and with a luminosity equal to that observed between 2 and $10 \mathrm{KeV}$. From the new fits of the UV spectra we find that 5-50 \% of the UV emission can be produced by the X-ray radiation. Having selected the UV-brightest objects of the sample and assuming the most conservative value for the $\mathrm{X}$-ray luminosity, this fraction represents only the lower limit of the reprocessed UV emission. More realistic values of the reprocessed UV component demand the study of the UV and X-ray variability.

\section{References}

1. Rokaki, E., Boisson, C., and Collin-Souffrin, S., 1992, A\&A 253, 57

2. Piccinotti G., Mushotzky R.F., Boldt E.A., et al., 1982 ApJ 253, 485

3. Rokaki, E., Collin-Souffrin, S. and Magnan C., 1993, A\&A 272, 8

T. J.-L. Courvoisier and A. Blecha: Multi-Wavelength Continuum Emission of AGN, 489.

(C) 1994 IAU. Printed in the Netherlands. 